\title{
New efficient gene promoters from Stellaria media for plant genetic engineering
}

\author{
L.N. Efremova, S.R. Strelnikova, R.A. Komakhin \\ All-Russia Research Institute of Agricultural Biotechnology, Moscow, Russia
}

DOI 10.18699/ICG-PlantGen2019-42

(c) Autors, 2019

* e-mail: recombination@iab.ac.ru

\begin{abstract}
In a previous study we found that Stellaria media plants are the source of effective pro-SmAMP1 and pro-SmAMP2 promoters for plant biotechnology. A low level of polymorphism between nucleotide sequences of these promoters makes it possible to identify point mutations resulting in differences in their properties. A high degree of identity between pro-SmAMP1 and pro-SmAMP2 impedes the simultaneous employment of both promoters in one genetic construct for plant transformation. To create novel regulatory elements, we cloned the promoters of the $a$-harpinine gene and of the defensine gene from chickweed. These novel promoters do not have a homology with any other known promoter and have the same or a greater efficiency as the CaMV35S promoter in transient expression.

Key words: promoters; gene expression; reporter genes; transient expression.
\end{abstract}

\section{Introduction}

At the present time in plant biotechnology there is a deficit of regulatory elements for effective expression of recombinant genes due, first, to the lack of native promoters of plant genes with necessary parameters (Naqvi et al., 2010) and, secondly, to complexity in using available promoters for coordinated transcriptional control (Shrestha et al., 2018). We had previously found that hevein-like peptide genes from chickweed (Stellaria media) are a source of strong constitutive promoters for biotechnology of cultivated plants (Shukurov et al., 2012; Komakhin et al., 2016; Vysotskii et al., 2016; Vetchinkina et al., 2016). E. g., in transient expression experiments in Nicotiana benthamiana pro-SmAMP1 and pro-SmAMP2 promoters were 2-4 times more effective than the CaMV35S promoter; in rape (Brassica napus) and sugar beet (Beta vulgaris) plants pro-SmAMP1 and pro-SmAMP2 were comparable to CaMV35S (Madzharova et al., 2018). The functionality of the pro-SmAMP2 promoter was shown in the calluses of flax (Linum usitatissimum). In the homozygous lines of transgenic tobacco (Nicotiana tabacum), the proSmAMP1 and pro-SmAMP2 promoters are twice as strong as the CaMV35S promoter. The both promoters are at least as effective as the duplicated CaMV35S promoter for neomycin phosphotransferase II (nptII) gene control in the selection of transgenic tobacco and Arabidopsis plants on media with the antibiotic kanamycin at recommended concentrations. In the present research we focused on the fact that the proSmAMP1 and pro-SmAMP2 promoters are $94 \%$ identical, but differ significantly in their properties in plant cells. In transient expression in $N$. benthamiana the deletion variant (-442 bp) of the pro-SmAMP1 promoter was approximately 1.5 times stronger than the deletion variant $(-455 \mathrm{bp})$ of the pro-SmAMP2 promoter. At the same time, in the medium with excess kanamycin $(350 \mathrm{mg} / \mathrm{L})$, the effectiveness of selection of tobacco cells with the use of pro-SmAMP1 was a third as high as with pro-SmAMP2.

\section{Materials and methods}

Plants for experiments comprised tobaccos (N. benthamiana (Domin) and N. tabacum L. cultivar Samsun-NN). The plants were grown at $16 / 8$-h illumination and $18-22{ }^{\circ} \mathrm{C}$. Cells of Agrobacterium tumefaciens strain GV3101 were used for infiltration, and strain AGL0, for genetic transformation. Agrobacterial strain GV2260/C58C1 with genetic construct pLH7000 containing the gene of 19 tobusvirus suppressor protein was used for the agroinfiltration of $N$. benthamiana plants to suppress RNA interference. The strains were injected into leaves with a needle-free syringe. Agrobacterial transformation of plants was performed by the "lawn" method, which had been used earlier (Komakhin et al., 2016). Genetic constructs for transient expression containing the reporter gene gus controlled by the plant promoters were incorporated into plasmid pCambia1381Z (Cambia, Australia). Genetic constructs for plant transformation containing the nptII gene controlled by the plant promoters were incorporated into plasmid pCambia2300 (Cambia, Australia).

\section{Results and discussion}

Our results suggest that 25 point mutations between the sequences of the pro-SmAMP1 and pro-SmAMP2 promoters (amounting to only $6 \%$ of their length) account for their different effectiveness in plant cells. However, it is not clear which of them are functional in the -455- and -442- bp deletion variants. Thus, to clarify the principles of functioning of regulatory elements, we first found minimal promoters comprising only cis-elements essential for functioning.

The search for minimal promoters was carried out via consecutive deletion analysis starting from the $5^{\prime}$ end avoiding fractures within cis-acting elements found in silico. As a result, nine new deletion variants from -443 to $-76 \mathrm{bp}$ in size were prepared for each promoter. We established that the minimal nucleotide sequences of both promoters are nearly 120-bp long and comprise the identical transcription start sites (TSS) and cis-element TATA box, CAAT box, G box and ABRE. The presence of the CAAT box, G box and ABRE is necessary for activity of both core promoters including the TATA box and TSS. Nucleotide sequences were found to be $94 \%$ identical and having differences in their properties. On shortening the sequence down to the minimal size, the effectiveness of pro-SmAMP1 in the transient expression of 


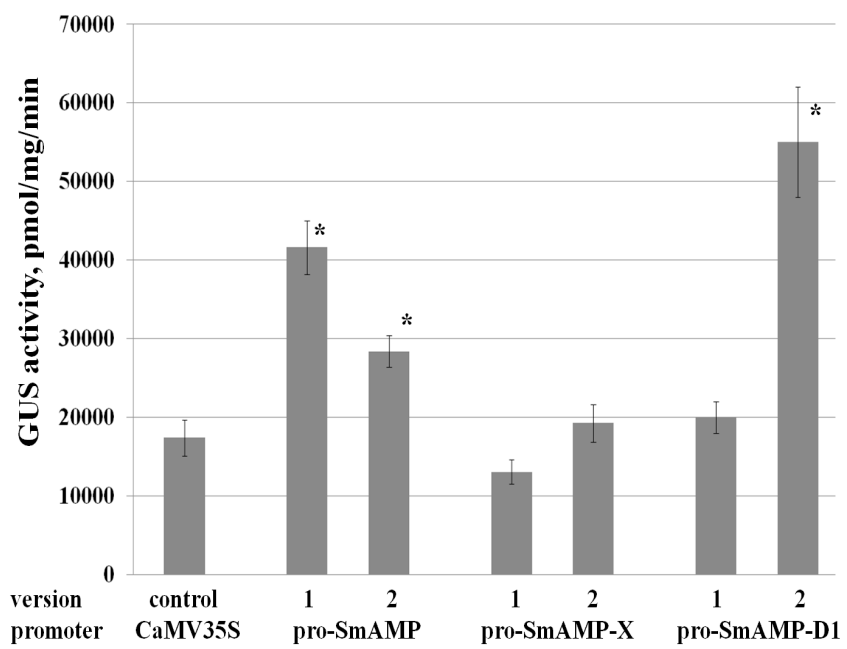

Figure 1. GUS activity in leaves of $N$. benthamiana plants at transient expression using plant promoters from S. media and virus CaMV35S promoter. Vertical lines indicate the standard error. Horizontal numbers show an individual version of plant promoters. ${ }^{*}$ values that are significantly different from the control at a $5 \%$ significance level. Gus activity was measured fluorimetrically, using 4-methylumbelliferyl-D-glucuronide (4-MU) as a substrate.

the gus gene in N. benthamiana plants somewhat increased, while in the case of pro-SmAMP2 it somewhat decreased, and the effectiveness ratio pro-SmAMP1 : pro-SmAMP2 reached 1.5-1.6. In the medium with excess kanamycin, the effectiveness of selection of transgenic tobacco cells with the use of nptII gene under control of pro-SmAMP1 was much lower than with pro-SmAMP2. In the case of minimal promoters, the differences in their properties are governed by only 7-9 point mutations; their functionality will be studied with a site-directed mutagenesis technique.

A significant similarity of nucleotide sequences in the promoters of hevein-like genes precludes their joint use within the same genetic construct to prevent recombination between the repeats. To create novel regulatory elements, we cloned the promoters of the $\alpha$-harpinine gene (pro-SmAMP-X) and defensine gene (pro-SmAMP-D1) from chickweed using the genome walking method (Pogorelko and Fursova, 2008). No homology of nucleotide sequences of the novel promoters, pro-SmAMP-X and pro-SmAMP-D1, with other known promoters was. In the chickweed genome, there are at least two versions of each new promoter, which are $83-93 \%$ identical. All new promoters are functional in the transient expression of the gus gene in $N$. benthamiana plants and are either as effective as virus promoter CaMV35S or substantially stronger than it (Figure 1).

As in the case with the promoters of the hevein-like peptide genes, point polymorphisms between two versions of the same promoter, pro-SmAMP-X and pro-SmAMP-D1, are functional and govern the difference in their effectiveness. In particular, one version of pro-SmAMP-D1 is approximately thrice stronger than the other one and the CaMV35S promoter.

\section{Conclusions}

Our results show that the gene promoters of various families of antimicrobial peptides from chickweed (S. media) are effective in transgenic plants for controling recombinant genes and may be successfully used in plant biotechnology. Lack of homology between different promoters provides the possibility of their simultaneous employment within a single genetic construct for plant transformation. Nucleotide polymorphism between the sequences of related promoters is functional and governs a different character of their activity in the cells of transgenic plants. Elucidation of the role of functional polymorphisms should pave the way for the production of new synthetic promoters featuring the best properties of native promoters.

\section{References}

Komakhin R.A., Vysotskii D.A., Shukurov R.R., Voblikova V.D., Komakhina V.V., Strelnikova S.R., Vetchinkina E.M., Babakov A.V. Novel strong promoter of antimicrobial peptides gene proSmAMP2 from chickweed (Stellaria media). BMC Biotechnology. 2016;16: 43. DOI 10.1186/s12896-016-0273-x.

Madzharova N.V., Kazakova K.A., Strelnikova S.R., Snycheva O.A., Vetchinkina E.M., Efremova L.N., Vysotskii D.A., Babakov A.V., Komakhin R.A. Promoters pro-SmAMP1 and pro-SmAMP2 from Wild Plant Stellaria media for the Biotechnology of Dicotyledons. Russ. J. Plant Physiol. 2018;65:750-761. DOI 10.1134/S1021443 718040040.

Naqvi S., Farre' G., Sanahuja G., Capell T., Zhu C., Christo P. When more is better: Multigene engineering in plants. Trends in Plant Science. 2010;15:48-56. https://doi.org/10.1016/j.tplants.2009.09.010

Shrestha A., Khan A., Dey N. cis-trans Engineering: Advances and Perspectives on Customized Transcriptional Regulation in Plants. Mol Plant. 2018;2;11(7):886-898. DOI 10.1016/j.molp.2018.05.008.

Shukurov R.R., Voblikova V.D., Nikonorova A.K., Komakhin R.A., Komakhina, V.V., Egorov, T.A., Grishin, E.V., Babakov A.V. Transformation of tobacco and Arabidopsis plants with Stellaria media genes encoding novel hevein-like peptides increases their resistance to fungal pathogens. Transgenic Res. 2012;21:313-325. https://doi. org/10.1007/s11248-011-9534-6.

Vetchinkina E.M., Komakhina V.V., Vysotskii D.A., Zaitsev D.V., Smirnov A.N., Babakov A.V., Komakhin R.A. Expression of plant antimicrobial peptide pro-SmAMP2 gene increases resistance of transgenic potato plants to Alternaria and Fusarium pathogens. Russ. J. Genet. 2016;52:939-951. https://doi.org/10.1134/S102279 5416080147.

Vysotskii D.A., Strel'nikova S.R., Efremova L.N., Vetchinkina E.M., Babakov A.V., Komakhin R.A. Structural and functional analysis of new plant promoter pro-SmAMP1 from Stellaria media. Russ. J. Plant Physiol. 2016;63:663-672. https://doi.org/10.1134/ S1021443716050174

Acknowledgements. The reported study was funded by RFBR according to the research project № 19-016-00067.

Conflict of interest. The authors declare no conflict of interest. 